\title{
Characterization of the Mechanical Properties of the Human Aortic Arch Using an Expansion Method
}

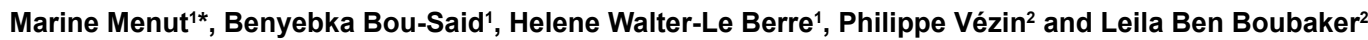

${ }^{1}$ Université de Lyon, INSA-Lyon, LaMCoS UMR5259, F-69621, France

${ }^{2}$ Université de Lyon, F-69622, Lyon, France ; Université Claude Bernard Lyon 1; IFSTTAR, UMR_T9406, LBMC Laboratoire de Biomécanique et Mécanique des Chocs, F69675, Bronoffoff

\begin{abstract}
Analyzing cardiovascular diseases leads to multidisciplinary problems which require transversal and complementary approaches. This study focuses on the identification of the mechanical properties of the aortic arch Stereo-correlation technique is used to measure the strain field in the aortic arch during its expansion. The aorta is immersed in water which allows better results in terms of measurement method and to take into account the residual stress and strain effects.

Results are obtained at different values of expansion: 9 samples are collected, 7 of them are frozen before the experiment and 2 are fresh. The mean age is 76 years old at the volunteers' time of death. 4 samples did not lead to conclusive results because of the quality of the arterial wall and leaks that happened during the experiment preventing a proper expansion. The horizontal and vertical displacements are relatively homogeneous for all the samples: two preferred radial and longitudinal directions are observed. The strain fields associated with these directions show heterogeneities and have significant differences between fresh and frozen specimen.

The final objective is to perform virtual surgical simulation of the whole endovascular stent graft procedure for an aortic aneurysm. This procedure has a high rate of short-term success and its indication compared to open surgery is increasing but it needs to be more reliable and secure. In this context, it is important to identify the mechanical properties of the aorta for further numerical simulations.
\end{abstract}

Keywords: Cardiovascular Diseases, Thoracic Aortic Aneurysm, Mechanical Properties, Stereo-correlation

\section{Introduction}

Cardiovascular diseases are the leading cause of death worldwide [1]. They lead to multidisciplinary problems that require diversity, transversal and complementary approaches. This study focuses on the thoracic aorta, precisely on the aortic arch in which the formation of an aneurysm needs an effective treatment. The aim is to identify the mechanical behavior of the aortic arch in order to provide a diagnostic tool and support for clinical planning.

There are various techniques to characterize mechanically the aorta: expansion tests [2], uniaxial tensile tests [3], biaxial tensile tests [4] and indentation methods [5]. These methods have shown the nonlinear anisotropic and viscoelastic nature of the aorta and the heterogeneous behavior between healthy and sick tissue.

Characterizing the nonlinear material behavior can be performed using stereo-correlation technique. This technique has already been applied successfully to biological soft materials for the human liver [6] or the aorta [7] characterization. It is a well-known method for the measurement of displacements and local deformations from series of speckled images. Unlike the global displacement methods, this noncontact method measures the actual displacement applied to the test sample [8], which may be different from the grip displacement, especially in case of sliding between the jaws.

In this study, stereo-correlation technique is used to measure the strain field in the aortic arch of a human aorta immersed in water. Immersion allows to have better results in terms of measurement method and to take into account the residual stress and strain effects [9].

\section{Material and Methods}

\section{Sample preparation}

For this study, 9 samples of aortas were collected from human subjects from the Department of Anatomy of the Faculty of Medicine of Lyon through the French voluntary corpse donation to Science program. The age of volunteers ranges from 50 to 96 years old. The mean age is 76 years old at the volunteers' time of death. There are 3 males and 6 females. 7 of these samples were frozen for later use while 2 are tested immediately after removal. They were cleaned with a saline solution so that only the thoracic aorta including the ascending aorta, the arch and descending aorta remained. 4 samples did not lead to conclusive results because of the quality of the arterial wall and leaks that happened during the experiment preventing a proper expansion.

Small arteries were tightened by several methods depending on their size. The arteries were closed by small pearls with the same diameter. These pearls were crushed to prevent any leaks. Sutures and glue were also used for arterioles. The sample was then speckled with a black water resistant spray paint which dries quickly to prevent the aorta from drying out, which could have altered the speckle.

${ }^{*}$ Corresponding author: Marine Menut, Universite de Lyon, INSA-Lyon, LaMCoS UMR5259, F-69621, France, Tel: 33604672008; E-mail: marine.menut@insa-lyon.fr

Received February 05, 2015; Accepted February 24, 2015; Published February 26, 2015

Citation: Menut M, Bou-Said B, Walter-Le Berre H, Vezin P, Ben Boubaker L (2015) Characterization of the Mechanical Properties of the Human Aortic Arch Using an Expansion Method. J Vasc Med Surg 3: 188. doi:10.4172/2329-6925.1000188

Copyright: ( 2015 Menut M, et al. This is an open-access article distributed under the terms of the Creative Commons Attribution License, which permits unrestricted use, distribution, and reproduction in any medium, provided the original author and source are credited. 
The sample was mounted on a support manufactured especially for this purpose after the installation of pipes at both ends. Carotid arteries and arterioles were attached to the support but were still free of movement. The aorta was not embedded on the support to leave the necessary space so that the expansion did not interact with it. Figure 1 shows the speckled sample on its support.

\section{Experimental installation}

The speckled aorta was immersed in a tank of a $125 \mathrm{~L}$ water capacity, heated to $37^{\circ} \mathrm{C}$ to duplicate as close as possible the physiological conditions (Figure 2). One of the main difficulties in the use of stereocorrelation technique on biological tissues is hydration. The sample surface shines and reflects light, causing the appearance of stray pixels preventing the correlation. When the aorta is immersed in water, we can obtain dull images which is an optimal situation for the correlation calculation.

Two high-speed Photron Fastcam SA3 ${ }^{\circ}$ digital video cameras with an image size of $1024 \times 1024$ pixels $^{2}$ were used to record the sample evolution during test. The acquisition frequency was $50 \mathrm{~Hz}$ to have the more light possible. Lens EXDG Sigma 27-70 mm were used and the aperture was $16 \mathrm{~mm}$, which gives a sufficient field depth for the aortic arch expansion. The camera locations were outside the tank perpendicular and close to the glass surface. Figure 2 shows the experimental installation including the aorta under water fixed to the support with the image acquisition cameras installed outside.

The pressure was measured with two pressure sensors EPB-PW3.5BS-/L3M installed inside the inlet pipe and the aortic arch for accurate pressure measurements. The measurement frequency was $50 \mathrm{~Hz}$. A trigger was used to synchronize the image acquisition and pressure sensors. An average flow rate of $5 \mathrm{~L} / \mathrm{min}$ was provided from a hydraulic pump and the aorta expansion was obtained thanks to an adjustable valve at the output. The experimental setup also measured the leaks through a displacement sensor present in the tank. The displacement field in the aortic arch was determined with Vic3D@ [10] software. Image correlation technique was applied to each image in order to get the displacements (along the aortic arch with $25 \times 25 \mathrm{px}^{2}$ patterns corresponding to $1.5 \times 1.5 \mathrm{~mm}^{2}$ ).

\section{Results on Displacement and Strain Calculation}

Figure 3 shows the image exploitation with Vic3D@ at different expansion levels for a frozen specimen: $\mathrm{x}$-axis and $\mathrm{y}$-axis correspond to the horizontal and vertical axis of Figure 4. It shows Lagrangian strain along the aortic arch. Figure $4 \mathrm{a}-\mathrm{c}$ show strain field examples at different values of expansion for a frozen aorta and two fresh aortas. The results are presented on an average $12 \times 12 \mathrm{~mm}^{2}$ grid. The horizontal and vertical displacements are relatively homogeneous while the strain fields show heterogeneities.

\section{Discussion}

Pressure inside the thoracic aorta varies between 133 mbar to 200 mbar for systolic and between 80 mbar to 133 mbar for the average diastole for the adult [11]. The expansion applied on the aorta and exploitable with Vic3D@ can reach 600 mbar which is enough for this study. The aim is to remain in the pressure range imposed by the heart pulsations to characterize the aortic tissue behavior during the stent deployment.

The expansion pressure was enough, considering that the final objective is not to reach aortic rupture, but to obtain its behavior during a surgical operation. The experimental results do not consider the mechanical behavior of each layer of the aorta but its overall behavior. Stereo-correlation results show "structured" heterogeneity of the strain field in the aortic arch, although the orthotropic nature of the aortic tissue is well established in the literature for healthy tissue [11]. These heterogeneities can be due either to the calcifications or to the freezing. The differences in behavior between frozen tissue and fresh tissue were repeatedly studied in the literature [12] which shows the difference in fiber orientation constituting the different layers of the aortic tissue [13]. For the obtained displacement fields, two preferential radial and longitudinal directions are observed. The strain fields associated to these directions are very different when comparing fresh and frozen samples: there was $15.2 \%$ mean expansion associated with an average 100 mbar pressure in the radial direction and $11.5 \%$ in the longitudinal direction for the 3 frozen samples. For the 2 fresh samples, there was 9.7\% mean expansion associated with 300 mbar pressure in the radial direction and $3.9 \%$ in the longitudinal direction. The frozen samples seem to have larger strain for smaller pressure. This can be due to the modification of collagen and elastin.

This study has some limitations. It requires small variations in terms of pressure to respect the linearity and the small deformation assumptions. Results were obtained from a small number of samples. Thus a statistical analysis would not be representative. Aortas were

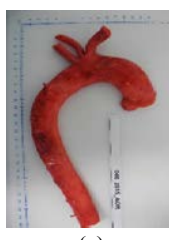

(a)

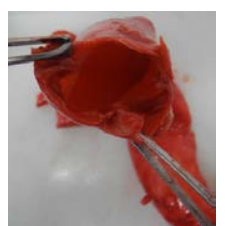

(b)

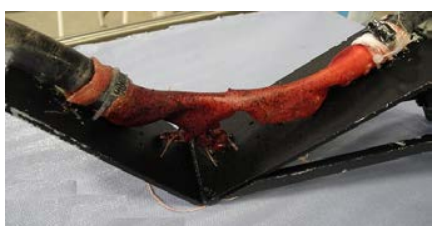

(c)
Figure 1: (a) Sample cleaned (b) Inside of the thoracic arch shows no sign of calcification for this fresh sample (c) Speckled sample installed on its support.

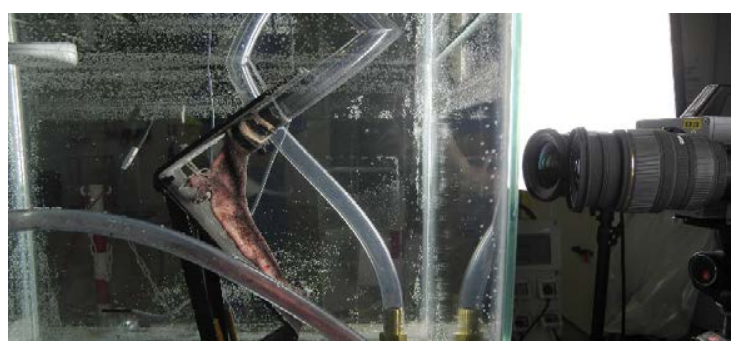

Figure 2: Aorta immersed in water before the experiment.

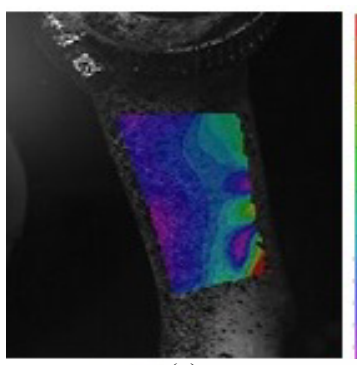

(a)

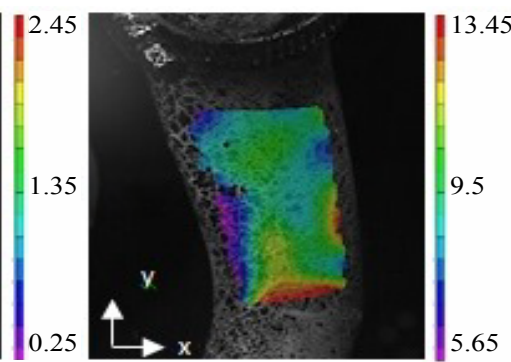

(b)
Figure 3: Lagrangian strains (\%) $1 \%$ mean (a) and 10\% mean (b). 

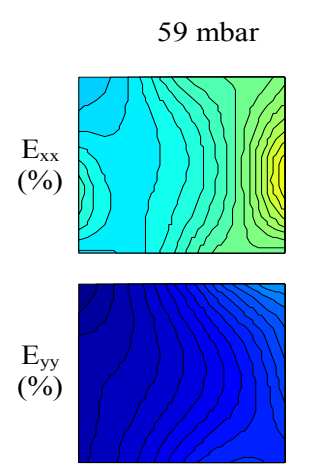

67 mbar
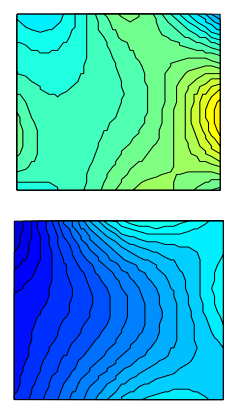

73 mbar
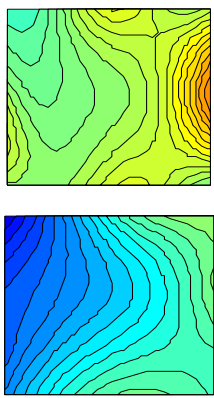

83 mbar
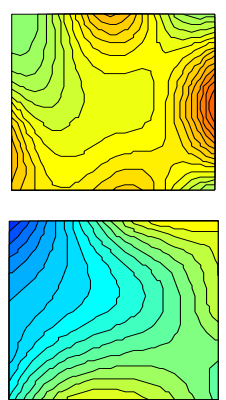

96 mbar
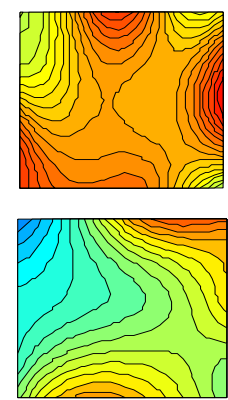

107 mbar

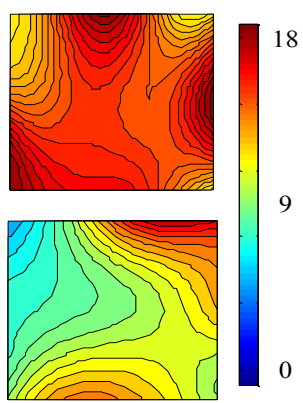

Figure 4a: Strain fields for different frozen aorta expansions : from $59 \mathrm{mbar}(44 \mathrm{~mm} \mathrm{Hg})$ to $107 \mathrm{mbar}(80 \mathrm{~mm} \mathrm{Hg})$. Horizontal and vertical axes are $\mathrm{x}$ and y orientations (Frozen aorta ( $F, 50$ years old)).

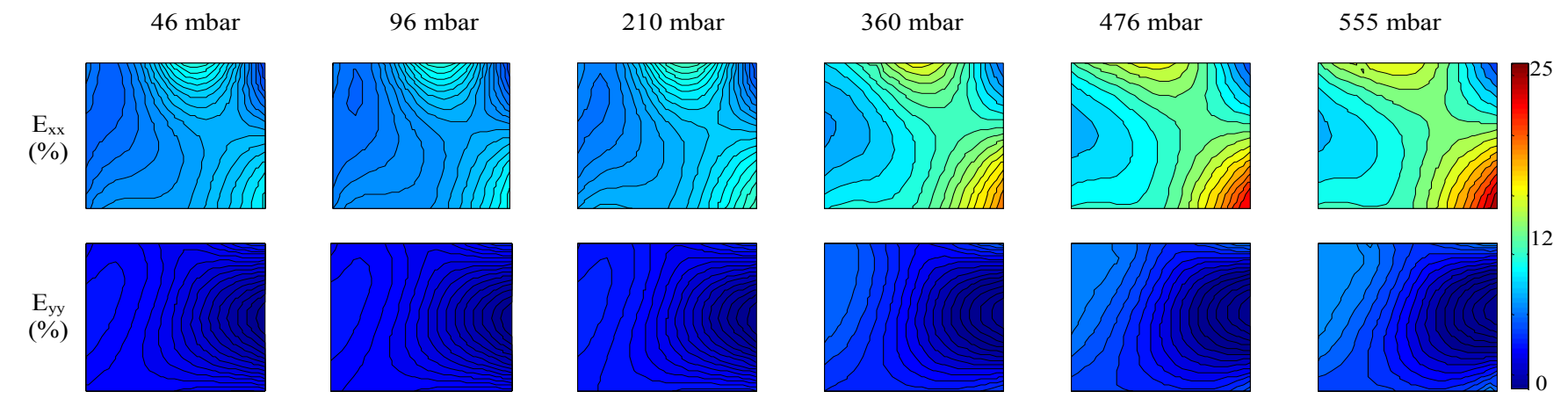

Figure 4b: Strain fields for different fresh aorta expansions : from $46 \mathrm{mbar}(33 \mathrm{~mm} \mathrm{Hg})$ to $555 \mathrm{mbar}(416 \mathrm{~mm} \mathrm{Hg})$. Horizontal and vertical axes are $\mathrm{x}$ and y orientations (Fresh aorta $(F, 89$ years old $)$ ).

77 mbar
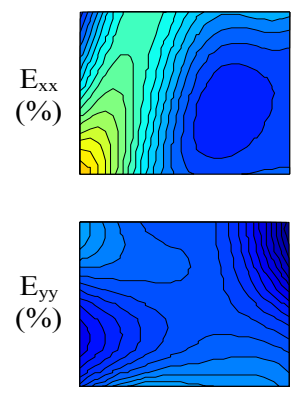

94 mbar
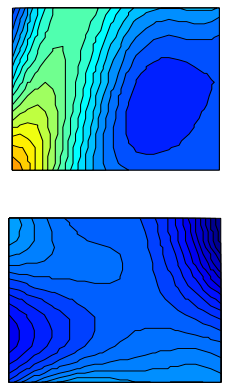

118 mbar
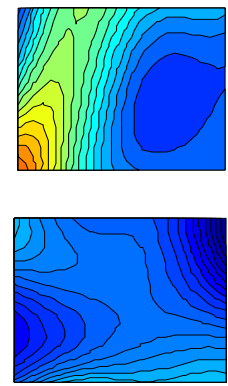

157 mbar
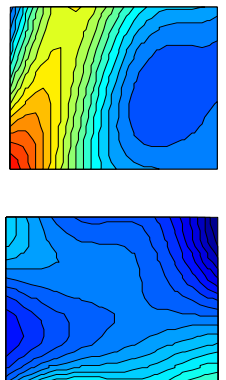

224 mbar
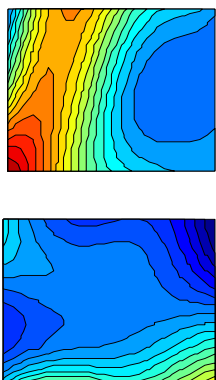

311 mbar

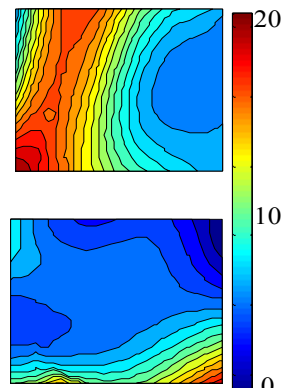

Figure 4c: Strain fields for differe
(Fresh aorta (F, 86 years old)).

collected on human subjects through the French voluntary corpse donation to Science program, which do not communicate information such as cause of death or health status. These factors may influence significantly the arterial tissue behavior.

\section{Conclusion}

The final objective of our project is to perform virtual surgical simulation of the whole endovascular stent graft procedure for an aortic aneurysm. This procedure has a high rate of short-term success and its indication compared to open surgery is increasing. Despite many benefits (reduced blood loss, reduced recovery time), the hindsight is insufficient and there are limitations related to complex anatomical configurations. This procedure therefore needs to be more reliable and secure. In this context, it is important to identify the mechanical behavior of the aorta for further numerical simulations.

This approach is part of a computer-aided design project for surgical procedures. It needs accurate tools / tissue interaction including blood behavior simulations to suggest a realistic customized solution for both a proper deployment system and a suitable stent recommendation.

\section{Ethical Declaration}

All tests and procedures were in line with the French ethical rules and the law that allows experiments involving post mortem human 
Citation: Menut M, Bou-Said B, Walter-Le Berre H, Vezin P, Ben Boubaker L (2015) Characterization of the Mechanical Properties of the Human Aortic Arch Using an Expansion Method. J Vasc Med Surg 3: 188. doi:10.4172/2329-6925.1000188

subjects (PMHS) for biomedical research through the Body Donation to Science program.

\section{References}

1. OMS (2011) Rapport sur la situation mondiale des maladies non transmissibles 2010. Genève.

2. Wang JJ (2006) Noninvasive determination of arterial pressure-dependent compliance in young subjects using an arterial tonometer. Biomed Eng Appl Basis Communic 18: 111-118.

3. Raghavan ML, Webster MW, Vorp DA (1996) Ex vivo biomechanical behavior of abdominal aortic aneurysm: assessment using a new mathematical model. Ann Biomed Eng 24: 573-582.

4. Vande Geest JP, Sacks MS, Vorp DA (2006) The effects of aneurysm on the biaxial mechanical behavior of human abdominal aorta. J Biomech 39: 13241334

5. Haigron P, Duménil A, Kaladji A, Rochettee M, Bou Said B, et al. (2013) Angiovision: Aortic stent-graft placement by augmented angionavigation. IRBM 34: $167-175$

6. Brunon A, Bruyère-Garnier K, Coret M (2011) Characterization of the nonlinear behaviour and the failure of human liver capsule through inflation tests. J Mech Behav Biomed Mater 4: 1572-1581.
7. Avril S, Badel P, Duprey A (2010) Anisotropic and hyperelastic identification of in vitro human arteries from full-field optical measurements. J Biomech 43: 2978-2985.

8. Zhang D, Charles D Eggleton, Dwayne D Arola (2002) Evaluating the mechanical behaviour or arterial tissue using digital image correlation. Experimental Mechanics 42: 409-416.

9. D Peterson, J Bronzino (2008) Biomechanical: Principales and Applications New York: CRS Press Taylor \& Francis Group.

10. Hubert Schreier, Jean-Jose Orteu, Michael A Sutton (2009) Image Correlation for Shape, Motion and Deformation Measurements: Basic Concepts, Theory and Applications. Springer Verlag.

11. Y Fung (1996) Biomechanics Circulation. New York, Springer-Verlag.

12. Adham M, Gournier JP, Favre JP, De La Roche E, Ducerf C, et al. (1996) Mechanical characteristics of fresh and frozen human descending thoracic aorta. J Surg Res 64: 32-34.

13. Flamini V, Kerskens C, Simms C, Lally C (2013) Fibre orientation of fresh and frozen porcine aorta determined non-invasively using diffusion tensor imaging. Med Eng Phys 35: 765-776. 